\title{
AKR1C3 inhibition therapy in castration-resistant prostate cancer and breast cancer: lessons from responses to SN33638
}

\author{
Wen Zhou ${ }^{1 *}$ and Patrizia Limonta ${ }^{2 *}$ \\ ' Braman Family Breast Cancer Institute, University of Miami Sylvester Comprehensive Cancer Center, University of Miami Miller School of Medicine, \\ Miami, FL, USA \\ 2 Department of Pharmacological and Biomolecular Sciences, Università degli Studi di Milano, Milan, Italy \\ *Correspondence: wzhou@med.miami.edu; patrizia.limonta@unimi.it \\ Edited and reviewed by: \\ Gabriella Castoria, II University of Naples, Italy
}

Keywords: AKR1C3, SN33638, castration-resistant prostate cancer, breast cancer, testosterone, 17 $\beta$-estradiol

\section{A commentary on}

The activity of SN33638, an inhibitor of AKR1C3, on testosterone and $17 \beta$ estradiol production and function in castration-resistant prostate cancer and ER-positive breast cancer

by Yin YD, Fu M, Brooke DG, Heinrich DM, Denny WA, Jamieson SMF. (2014). Front Oncol 4:159. doi:10.3389/fonc.2014.00159

In the current issue of Frontiers in Oncology - Cancer Endocrinology Section, Yin et al. report that the aldo-keto reductase family 1 member C3 (AKR1C3) inhibitor SN33638 was able to reduce testosterone production, PSA expression, and cell proliferation only in some castration-resistant prostate cancer (CRPC) cells, overexpressing the enzyme, but not the majority of a panel of prostate and breast cell lines investigated, and revealing a novel caveat in developing AKR1C3 inhibitors for cancer therapy.

Prostate and breast cancers are the most common cancer and the second-leading cause of cancer-related deaths in males and females, respectively $(1,2)$. The mainstay treatment for advanced or metastatic prostate or ER-positive breast carcinoma is androgen-deprivation therapy or antiestrogen therapy. Although initially these treatments are beneficial and reduce tumor burden, tumors ultimately become resistant to these therapies. There are few treatment options for this stage of cancers (termed CRPC and tamoxifen-resistant breast cancer). Thus, new approaches to treat or prevent these hormone-insensitive cancers or their progression are in great need.

The AKR1C3, also known as $17 \beta-$ hydroxysteroid dehydrogenase type 5 , is capable to produce intratumorally testosterone and $17 \beta$-estradiol by reducing the androgen precursors and estrone, respectively. The local conversion of less potent hormones to more potent ones will lead to nuclear receptor activation and tumor progression. Therefore, AKR1C3 has recently been regarded as a potential anti-cancer drug target in both CRPC and ER-positive breast cancer $(3,4)$.

The AKR1C3 inhibitor SN33638 is a lead compound newly synthesized by Dr. Jamieson's group (5). It had low nanomolar potency against AKR1C3 and >300fold selectivity for AKR1C3 over the other AKR1C isoforms. Equipped by this powerful compound, Yin et al. (6) treated a panel of CRPC and ER-positive breast cancer cell lines, in the presence of hormone or prostaglandin precursors, prior to evaluation of cell proliferation and lev-

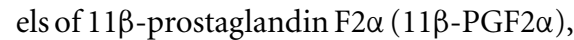
testosterone production, PSA expression, and cell proliferation (6). They observed that AKR1C3 mRNA was upregulated in CRPC in a meta-analysis of patient samples. $11 \beta-P G F 2 \alpha$ and testosterone levels in the cell line panel correlated with AKR1C3 protein expression. SN33638 prevented $11 \beta$-PGF2 $\alpha$ formation in cell lines that expressed AKR1C3, but only partially inhibited testosterone formation and subsequent cell proliferation and/or PSA expression only in high (LAPC4 AKR1C3) or moderate (22RV1) AKR1C3-expressing cell lines. This may be explained by that there is AKR1C3-independent steroid hormone production in most cells, and the data suggest that AKR1C3 is not an effective drug target in most CRPC cancer patients.

For ER-positive breast cancers, their finding on AKR1C3 inhibition is even less promising. They observed AKR1C3 mRNA was downregulated in patient samples, a poor correlation between AKR1C3 protein expression and $17 \beta$-estradiol production, while SN33638 was largely ineffective at preventing estrone reduction or cell proliferation. These results call into question of the possibility of using AKR1C3 inhibitors in treating breast cancer patients.

In summary, Yin et al. (6) revealed a novel finding that AKR1C3 inhibitor therapy is unlikely to be beneficial for the treatment of most CRPC and ER-positive breast cancers. Nevertheless, given the role of AKR1C3 in intratumoral steroidogenesis, we still hold enthusiastically the hope that a subpopulation of CRPC patients with high AKR1C3 might benefit from AKR1C3 inhibitor therapy, whose tumors are "addicted" from AKR1C3 to produce testosterone. The study by Yin et al. (6) provides a valuable framework for future preclinical or clinical studies aiming at verifying this hypothesis that AKR1C3 inhibition suppresses tumor formation in a selected population of AKR1C3-high CRPC patients.

\section{ACKNOWLEDGMENTS}

We thank Drs. Joyce Slingerland and Gabriella Castoria for comments and suggestions. 


\section{REFERENCES}

1. Feldman BJ, Feldman D. The development of androgen-independent prostate cancer. Nat Rev Cancer (2001) 1(1):34-45. doi:10.1038/ 35094009

2. Zhou W, Slingerland JM. Links between oestrogen receptor activation and proteolysis: relevance to hormone-regulated cancer therapy. Nat Rev Cancer (2014) 14(1):26-38. doi:10.1038/nrc3622

3. Montgomery RB, Mostaghel EA, Vessella R, Hess DL, Kalhorn TF, Higano CS, et al. Maintenance of intratumoral androgens in metastatic prostate cancer: a mechanism for castration-resistant tumor growth. Cancer Res (2008) 68:4447-54. doi:10.1158/ 0008-5472.CAN-08-0249

4. Sasano H, Miki Y, Nagasaki S, Suzuki T. In situ estrogen production and its regulation in human breast carcinoma: from endocrinology to intracrinology. Pathol Int (2009) 59:777-89. doi:10.1111/j.14401827.2009.02444.x
5. Heinrich DM, Flanagan JU, Jamieson SM, Silva S, Rigoreau LJ, Trivier E, et al. Synthesis and structure-activity relationships for 1-(4-(piperidin-1-ylsulfonyl)phenyl)pyrrolidin2-ones as novel non-carboxylate inhibitors of the aldo-keto reductase enzyme AKR1C3. Eur J Med Chem (2013) 62:738-44. doi:10.1016/j.ejmech.2013.01.047

6. Yin YD, Fu M, Brooke DG, Heinrich DM, Denny WA, Jamieson SMF. The activity of SN33638, an inhibitor of AKR1C3, on testosterone and $17 \beta$ estradiol production and function in castrationresistant prostate cancer and ER-positive breast cancer. Front Oncol (2014) 4:159. doi:10.3389/fonc. 2014.00159

Conflict of Interest Statement: The authors declare that the research was conducted in the absence of any commercial or financial relationships that could be construed as a potential conflict of interest.

Received:06 June 2014; accepted:06 June 2014; published online: 23 June 2014.

Citation: Zhou W and Limonta P (2014) AKR1C3 inhibition therapy in castration-resistant prostate cancer and breast cancer: lessons from responses to SN33638. Front. Oncol. 4:162. doi: 10.3389/fonc.2014.00162

This article was submitted to Cancer Endocrinology, a section of the journal Frontiers in Oncology.

Copyright () 2014 Zhou and Limonta. This is an openaccess article distributed under the terms of the Creative Commons Attribution License (CC BY). The use, distribution or reproduction in other forums is permitted, provided the original author(s) or licensor are credited and that the original publication in this journal is cited, in accordance with accepted academic practice. No use, distribution or reproduction is permitted which does not comply with these terms. 